\title{
Sinus Bradycardia, CTCAE
}

National Cancer Institute

\section{Source}

National Cancer Institute. Sinus Bradycardia, CT CAE. NCI Thesaurus. Code C54940.

A disorder characterized by a dysrhythmia with a heart rate less than 60 beats per minute

that originates in the sinus node. 